\title{
Nanocomposites of Polyaniline and Cellulose Nanocrystals Prepared in Lyotropic Chiral Nematic Liquid Crystals
}

\author{
Dawei Zhang, ${ }^{1}$ Lihong Zhang, ${ }^{1}$ Bingzhe Wang, ${ }^{1}$ and Guangzhe Piao ${ }^{1,2,3}$ \\ ${ }^{1}$ School of Polymer Science and Engineering, Qingdao 266042, China \\ ${ }^{2}$ Key Laboratory of Rubber-Plastics of Ministry of Education, Qingdao 266042, China \\ ${ }^{3}$ Shandong Provincial Key Laboratory of Rubber-Plastics, Qingdao 266042, China \\ Correspondence should be addressed to Guangzhe Piao; piao@qust.edu.cn
}

Received 25 January 2013; Revised 15 March 2013; Accepted 20 March 2013

Academic Editor: Ram Katiyar

Copyright (C) 2013 Dawei Zhang et al. This is an open access article distributed under the Creative Commons Attribution License, which permits unrestricted use, distribution, and reproduction in any medium, provided the original work is properly cited.

\begin{abstract}
Stable lyotropic chiral nematic liquid crystals $\left(\mathrm{N}^{*}-\mathrm{LCs}\right.$ ) of cellulose nanocrystals $(\mathrm{CNs})$ were prepared via hydrolysis using sulfuric acid. The lyotropic $\mathrm{N}^{*}$-LCs were used as an asymmetric reaction field to synthesize polyaniline (PANI) onto CNs by in situ polymerization. As a primary step, we examined the mesophase transition of the $\mathrm{N}^{*}$-LCs of CNs suspension before and after in situ polymerization of aniline (ANI) by polarizing optical microscopy. The structure of nanocomposites of PANI/CNs was investigated at a microscopic level using Fourier transform infrared spectroscopy and X-ray diffraction. Influence of the CNs-to-ANI ratio on the morphology of the nanocomposites was also investigated at macroscopic level by scanning electron and transmission electron microscopies. It is found that the weight ratio of CNs to aniline in the suspension significantly influenced the size of the PANI particles and interaction between CNs and PANI. Moreover, electrical properties of the obtained PANI/CNs films were studied using standard four-probe technique. It is expected that the lyotropic $\mathrm{N}^{*}$-LCs of CNs might be available for an asymmetric reaction field to produce novel composites of conjugated materials.
\end{abstract}

\section{Introduction}

Conductive polymers have been extensively investigated and widely used in such products as electrolytic capacitors and secondary batteries [1]. Polymers have become essential for lightweight, high performance batteries used in notebook computers, cellular phones, and other portable equipment. Conductive polymers are also being studied for their use as materials in molecular devices, called the ultimate electronic devices [2]. Conductive polymers offer the promise of achieving next-generation displays and energy sources [3]. Thus, although many conductive polymers have been developed for various applications, polyaniline (PANI) has received great attention due to its simple and facile synthesis, good environment stability, and controllability.

Nanocomposites of conducting polymer and cellulose have attracted much attention because it has recently been shown that it is possible to manufacture redox polymer-based electrodes and batteries with high capacities and very good recycling performances [4-6].

Cellulose is the most abundant and renewable biopolymer in the world, which is widely distributed in many higher plants, some marine animals (e.g., tunicates), algae, fungi, bacteria, and so on, so it is considered as a prime candidate for replacing oil-based feedstock and an almost inexhaustible source of the raw material for the increasing demand of environmentally friendly and biodegradable products [7-9]. The higher plants such as cotton particularly possess high cellulose content and the CNs of the plants with diameters in the range of 5-20 nm and aspect ratio of about 1 to 100 times are commonly produced through acid hydrolysis $[10$, 11]. The CNs possess several advantages such as low cost, low density, nontoxicity, renewable nature biodegradability, and in particular they can form stable lyotropic $\mathrm{N}^{*}$-LCs phase above a critical concentration [12-14]. It has been reported that the $\mathrm{N}^{*}$-LCs phase was used as a hard template to synthesize other new materials with chiral nematic 
structures $[15,16]$. In this thesis, we attempted to prepare novel nanocomposites of conductive polymer and CNs in the cellulose $\mathrm{N}^{*}$-LCs reaction field for in situ asymmetric polymerization.

The composites of PANI and cellulose have been received great attention in recent years due to their interdisciplinary character with new properties and applications [17-19]. Yin et al. [17] prepared the PANI-cellulose composites using microcrystalline cellulose extracted from corn straw powder as raw material, and mainly investigated the electrical properties of the composites activated with various acids. Mattoso et al. [18] successfully produced electrically conductive nanocomposites made from PANI and cellulose nanofibrils (CNFs) by the in situ polymerization of aniline onto CNF. Shi et al. [19] developed a new route to construct supramolecular complex of PANI and cellulose through the noncovalent interaction. The composite films displayed highly homogeneous structure and improved mechanical properties as a result of good miscibility between PANI and cellulose. The electrical conductivity of the composite films could be enhanced significantly via doping of acid and the carbon black. Akagi [15] reported the polymerization of acetylene in an asymmetric reaction field constructed with chiral nematic LCs and showed that polyacetylene films formed from helical chains and fibrils can be synthesized. However, as far as we know, the systematic research on the preparation of the nanocomposites of PANI and CNs in the lyotropic $\mathrm{N}^{*}$-LCs reaction field is rarely exploited. The purpose of this study is to prepare stable lyotropic $\mathrm{N}^{*}$-LCs of CNs to be used as an asymmetric LC reaction field to synthesize PANI onto CNs by in situ polymerization. As a primary step, we examined the mesophase transition of the $\mathrm{N}^{*}$-LCs of CNs suspension before or after in situ polymerization of aniline by polarizing optical microscopy (POM). The structure of nanocomposites of PANI/CNs was investigated at a microscopic level using Fourier transform infrared spectroscopy and X-ray diffraction. Influence of the CNs-to-aniline ratio on the morphology of the nanocomposites was also investigated at macroscopic level by scanning electron and transmission electron microscopies. Moreover, electrical properties of the obtained PANI/CNs films were studied using standard fourprobe technique.

\section{Experimental}

2.1. Preparation of CNs Suspension. CNs suspension was prepared by acid hydrolysis according to $[10,11]$. The cotton cellulose (Whatman CF11) was mixed with $64 \mathrm{wt} \%$ sulfuric acid, stirred at $45^{\circ} \mathrm{C}$ for $3 \mathrm{~h}$, and diluted with cold distilled water to stop the reaction. Then the acid was removed through centrifugation and prolonged dialysis with distilled water until the PH outside dialysis bag was neutral. The sample thus obtained was concentrated by osmotic compression using dialysis bag with molecular weight cutoffs of 14000 and a 15 wt $\%$ poly (ethylene glycol) $\left(M_{w}=20000\right)$ solution, and the nanocrystal aggregates were disrupted by sonication about 10 minutes under ice-water bath. The cellulose suspension with desired concentration was obtained through dilution with distilled water. The concentration of the sample suspension was measured gravimetrically before and after evaporation of the water.

2.2. Preparation of PANI/CNs Nanocomposites. $1 \mathrm{M}$ aqueous hydrochloric acid $(\mathrm{HCl})$ solution was prepared by adding concentrated $\mathrm{HCl}$ to distilled water under stirring, and cooled about $0^{\circ} \mathrm{C}$ in freezer. Then $0.25 \mathrm{~g}$ ammonium peroxydisulfate (APS) and $0.1 \mathrm{~mL}$ aniline (ANI) were dissolved in $50 \mathrm{~mL}$ and $67 \mathrm{~mL}$ of $1.0 \mathrm{M} \mathrm{HCl}$ solution, respectively. The $\mathrm{APS} / \mathrm{HCl}$ solution was added to the $50 \mathrm{~mL}$ of CNs suspension. Then the above mixture was brought to the desired temperature and $\mathrm{ANI} / \mathrm{HCl}$ solution was added to start the polymerization of ANI. The polymerization was carried out by using magnetic stirring in ice-water bath. Before the start of the polymerization, the prepared suspension exhibited a white color. However, the color of this suspension turned gradually from white into blue, at last into dark green and stabilized thereafter. The dark green is the characteristic color of PANI in emeraldine oxidation state. We removed the excessive $\mathrm{HCl}$, APS, and byproducts through centrifugation and dialysis for 3 days. The nanocomposites suspensions with different concentrations were diluted with distilled water and dispersed by sonication. The nanocomposites films were prepared by casting the asdoped PANI/CNs suspensions onto microscope glass slides and dried at room temperature for $48 \mathrm{~h}$. The thickness of the dried conducting polymer composites ranged from 0.05 to $0.1 \mathrm{~mm}$ depending on the polyaniline concentration relative to CNs.

The structure and morphologies were characterized by using scanning electron microscope (SEM, JEOL, JSM6700F), transmission electron microscope (TEM, JEOL, JEM-2100), and X-ray diffraction (XRD, Rigaku Corporation, D/MAX-2500/PC). All samples were not stained. The ultraviolet-visible (UV-Vis) spectra of the nanocomposites in doped and dedoped states were recorded by UV-Vis spectrophotometer (SHIMADZU, UV-2400PC). Fourier transform infrared (FT-IR) spectra of the PANI/CNs nanocomposites, PANI, and cellulose were recorded with a FT-IR spectrometer (Bruker Corporation, VERTEX70) via grinding the film thoroughly with $\mathrm{KBr}$ powder and pressing into pellets. The optical textures of the suspensions of CNs and PANI/CNs were observed under a polarizing optical microscope (POM, Jiangnan Optics XP213) equipped with digital camera. The conductivities of the PANI/CNs films were measured by the four-probe technique and each sample was measured five times and the average value was taken.

\section{Results and Discussion}

Figure 1 shows POM images of $\mathrm{CNs}$ and PANI/CNs suspensions. A fingerprint texture characteristic of $\mathrm{N}^{*}$-LCs phase was observed (Figure 1(a)). The distance between the striae corresponds to a half helical pitch of the $\mathrm{N}^{*}$-LCs. Note that as the concentration of the CNs in the suspensions increases, the helical pitch observed in POM decreases gradually. The pitch was about $15 \mu \mathrm{m}$, which is consistent with the reported results [11]. After in situ polymerization of ANI in the $\mathrm{N}^{*}$-LCs, the finger-print texture cannot be observed by POM, as shown in Figure 1(b). But the fingerprint texture still maintained 


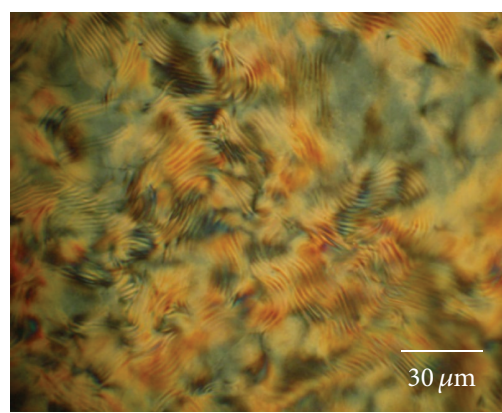

(a)

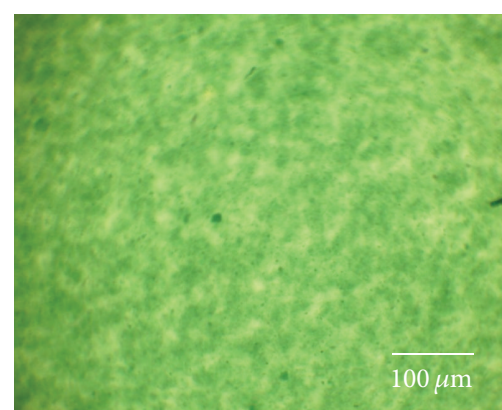

(b)

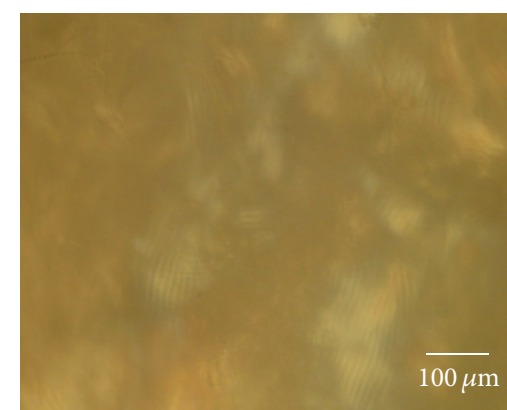

(c)

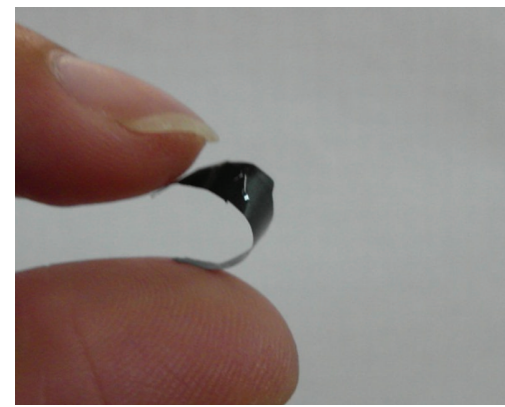

(d)

FIGURE 1: POM images of cellulose suspension after hydrolysis (a), suspension of PANI/CNs nanocomposites (b), cellulose suspension added a drop of aniline/ $\mathrm{HCl}(\mathrm{c})$, and photograph of a flexible film of PANI/CNs doped with $\mathrm{HCl}(\mathrm{d})$.

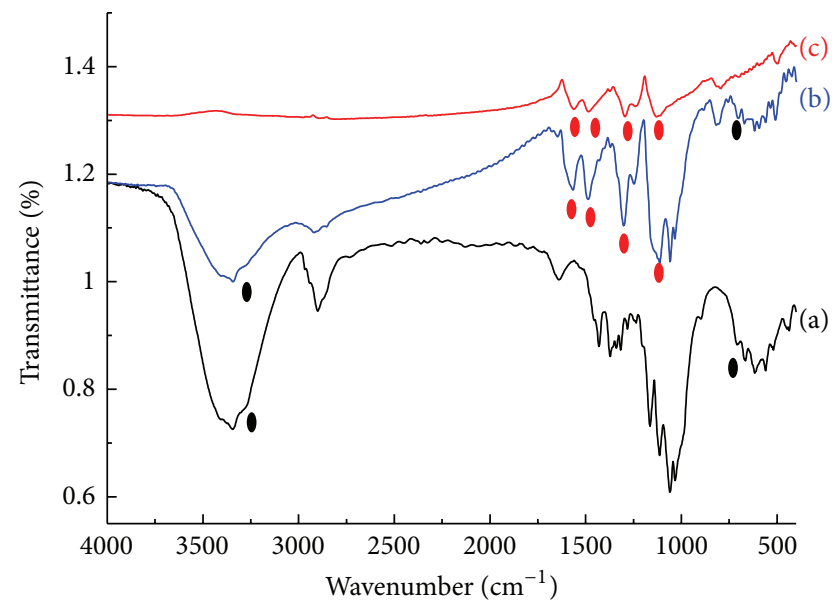

FIgURE 2: FT-IR spectra of cellulose (a), PANI particles (b), and PANI/CNs nanocomposites (c).

after a drop of $\mathrm{ANI} / \mathrm{HCl}$ solution was added in the $\mathrm{N}^{*}$-LCs suspension for several days, and the pitch became smaller than $7 \mu \mathrm{m}$ (Figure 1(c)). This is probably because the $\mathrm{N}^{*}$ LCs may be depressed by the introduction of "impurities," namely, the monomer, catalyst, or the resulting polymer, so the domain size of the chiral nematic phase decreased. The films of PANI/CNs, which are reasonably flexible and strong, were produced in doped state using $\mathrm{HCl}$ as shown in Figure 1(d).

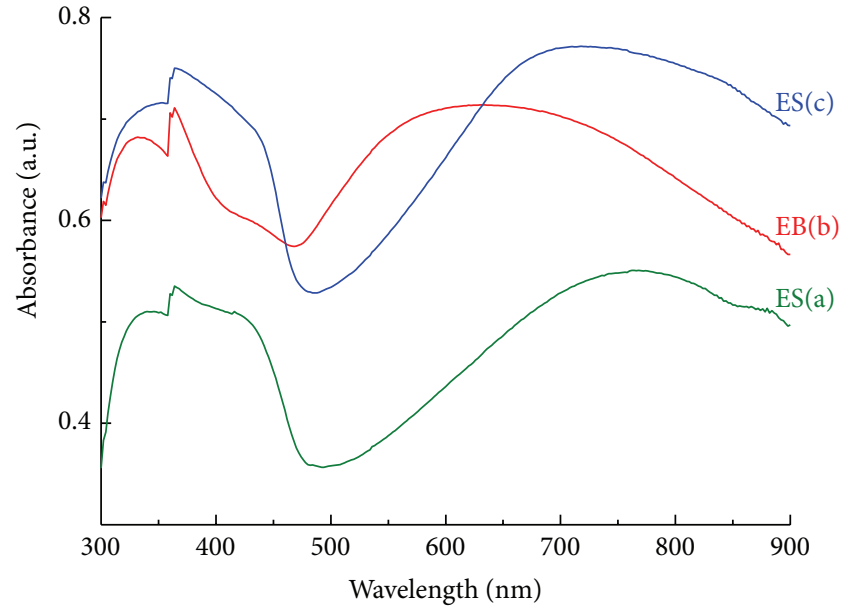

FIgURE 3: UV-Vis spectra of PANI/CNs nanocomposites: (a) as prepared in aqueous $\mathrm{HCl}$ solution, (b) dedoped with $\mathrm{NH}_{3}$, and (c) doped with $\mathrm{HCl}$.

The FT-IR spectra of cellulose, PANI, and PANI/CNs film are shown in Figure 2. The peaks near 3411, 3270, 2900, $1060,710 \mathrm{~cm}^{-1}$ in Figure 2(a) were associated with cellulose. A broad band at $3411 \mathrm{~cm}^{-1}$ was assigned to the stretching of hydroxyl groups. The peaks at 2900 and $1060 \mathrm{~cm}^{-1}$ arose from the $\mathrm{C}-\mathrm{H}$ stretching, and the $\mathrm{C}-\mathrm{O}-\mathrm{C}$ pyranose ring skeletal vibration, respectively. The peaks at 3270 and $710 \mathrm{~cm}^{-1}$ were attributed to the $I_{\beta}$ phase of cellulose. Figure 2(b) was the FT-IR spectrum of PANI in emeraldine oxidation 


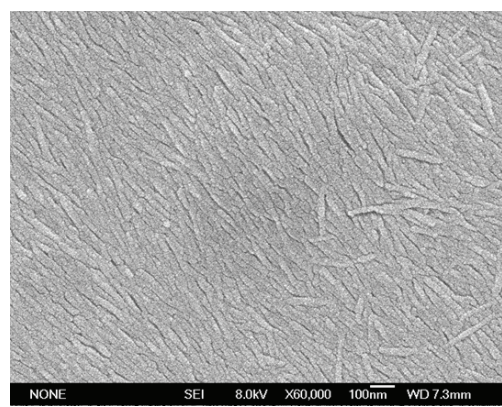

(a)

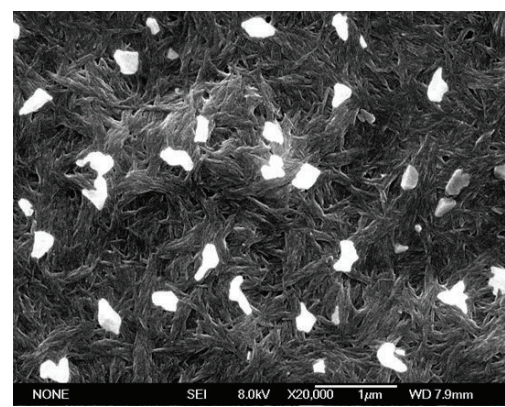

(b)

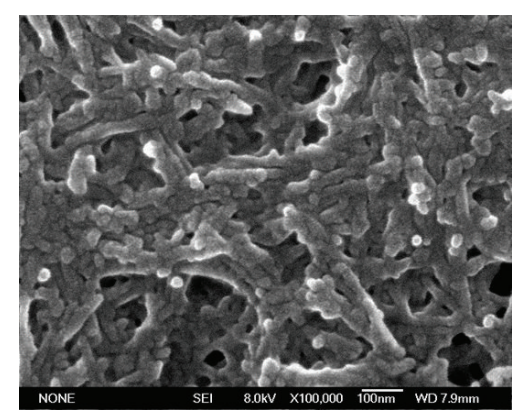

(c)

Figure 4: SEM images of CNs (a) and PANI/CNs nanocomposites ((b) and (c)). The CNs-to-ANI ratio by weight in the suspension is 5.69 (a) and 56.9(b).

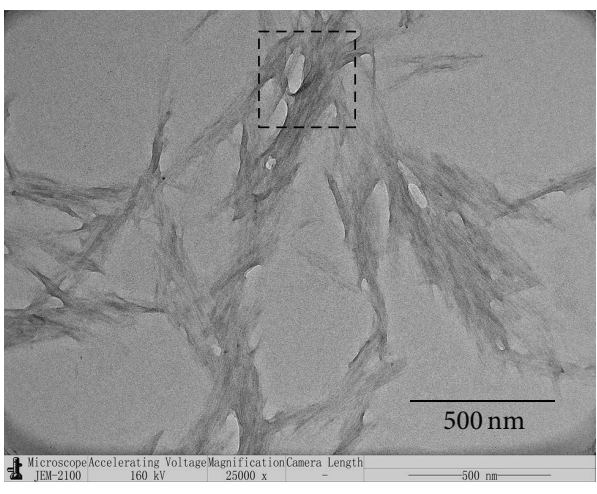

(a)

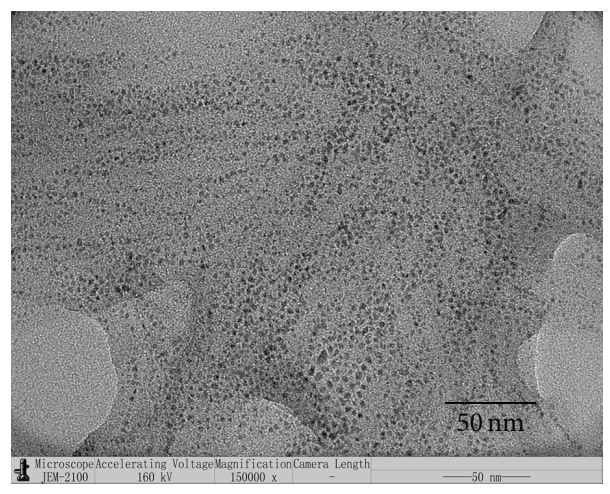

(b)

FIgURe 5: TEM images of PANI/CNs nanocomposites at the CNs-to-ANI ratio of 56.9. Part (b) is local amplification of part (a).

state. The peaks of 1566 and $1487 \mathrm{~cm}^{-1}$ originated from the stretching vibration of $\mathrm{N}=\mathrm{Q}=\mathrm{N}$ and $\mathrm{N}-\mathrm{B}-\mathrm{N}$ structures, respectively ( $\mathrm{B}$ and $\mathrm{Q}$ represent benzenoid and quinoid moieties in the PANI chains), and the peaks at 1299 and $1140 \mathrm{~cm}^{-1}$ were assigned to the stretching of the C-N band and the aromatic $\mathrm{C}-\mathrm{H}$ in-plane bending. Moreover, these characteristic peaks of CNs and PANI were also found in those of the PANI/CNs composites. The results indicated that the in situ polymerization of ANI in the $\mathrm{N}^{*}$-LCs still retained their chemical structures well.

The UV-Vis spectra of the doped and dedoped forms of the PANI/CNs nanocomposites showed that after dedoping the peak of excitonic transition was shifted from $622 \mathrm{~nm}$ to $764 \mathrm{~nm}$ as shown in Figures 3(a) and 3(b), indicating higher electronic transition energy. After redoping the sample with $\mathrm{HCl}$, it is found that the peaks were similar to those in original suspension, and this confirmed that this procedure between doping state and dedoping state was reversible.

The influence of the ratio of CNs to ANI by weight on the structure and morphologies of the PANI/CNs nanocomposites was investigated by SEM, as shown in Figure 4. As shown in Figure 4(a), the length of rod-like CNs ranges from 100 to $200 \mathrm{~nm}$ and width average is $20 \mathrm{~nm}$; the axial ratio is 5 to 10 . All of the nanofibrils form a nematic phase which performs as the finger-print texture on the microdomain. And after polymerization, the micrometer-scale domain of the nematic phase decreased to nanometer-scale. It is very interesting to note that the ratio of CNs to ANI significantly affected the size of PANI particles and interaction between PANI and CNs. The size of PANI particles was about $200 \mathrm{~nm}$ and the PANI particles were separated from CNs surface when the ratio of CNs to ANI was 5.69 (Figure 4(b)), while the PANI particles were significantly decreased to about $20 \mathrm{~nm}$ in size and absorbed on the surface of CNs when the ratio of CNs to ANI was 56.9 (Figure 4(c)). The hydrogen bands between hydroxyl groups of CNs and amine groups of aniline might serve as a traction force to assist the growing of the PANI over cellulose and avoid the large-scale aggregate formation [20]. The increase in the ratio of CNs to ANI prevented the primary aggregation of PANI particles and the CNs acted as the separant and dispersant during the in situ polymerization of ANI which eventually decreased the PANI size [21].

In order to further prove the previous speculation, we used TEM to characterize the PANI/CNs nanocomposites. It is observed from Figure 5(a) that rod-like CNs are about $200 \mathrm{~nm}$ in length and about $20 \mathrm{~nm}$ in width, which is consistent with the SEM results. It is surprising that much smaller particles about $3 \mathrm{~nm}$ existed on the surface of CNs, as shown in Figure 5(b). According to the previous work [2224], they might be PANI nanoparticles. It is observed that 
TABLE 1: Electrical conductivities of PANI/CNs films.

\begin{tabular}{lccc}
\hline Sample & CNs-to-ANI weight ratio & Resistivity, $\mathrm{K} \Omega \cdot \mathrm{cm}$ & Conductivity, S/cm \\
\hline 1 & 5.7 & $1.0 \times 10^{-1}$ & $1.0 \times 10^{-2}$ \\
2 & 11 & $3.6 \times 10^{-1}$ & $2.8 \times 10^{-3}$ \\
3 & 20 & $3.3 \times 10^{-1}$ & $3.0 \times 10^{-3}$ \\
4 & 28 & $6.4 \times 10^{-1}$ & $1.6 \times 10^{-3}$ \\
5 & 57 & $1.3 \times 10^{2}$ & $7.8 \times 10^{-5}$ \\
\hline
\end{tabular}

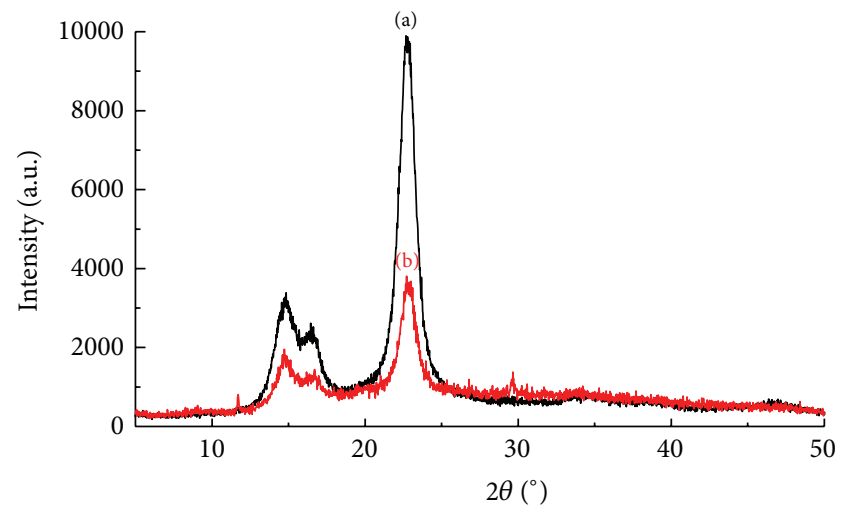

FIGURE 6: XRD patterns of CNs film (a) and PANI/CNs film (b), respectively.

PANI particles deposited on the surface of the CNs with little PANI aggregates especially in the optimized reaction condition. With weight being lighter, smaller PANI particles are much easier to be absorbed on the surface of cellulose. This demonstrated that the nanofibrils work as a good template for polymerization.

Figure 6 shows the crystal structure of $\mathrm{CNs}$ and the PANI/CNs films by XRD. Both films exhibited three peaks at

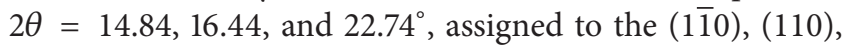
(200) planes of crystalline cellulose $I_{\beta}$, respectively [25]. It also indicated that the crystallinity of composite film was lower than that of CNs film. This could be because of the amorphous PANI and the association of PANI and CNs.

Conductivities as high as $10^{-2} \mathrm{~S} / \mathrm{cm}$ were obtained for the PANI/CNs films which belonged to semiconductor, as shown in Table 1, and the conductivity almost decreased with increase of the CNs-to-ANI ratio. The results were consistent with the reported one for PANI and cellulose nanofibrils, [18] when the polymerization was carried out under diluted conditions similar to those used in the present work.

\section{Conclusions}

The PANI/CNs nanocomposites were prepared for the first time by using the in situ polymerization with the lyotropic $\mathrm{N}^{*}$-LCs of CNs. UV-Vis spectra of the PANI/CVs nanocomposites verified the PANI being in the doped emeraldine salt form. It is found that the PANI particle size and conductivity of the nanocomposites decreased with increase of CNs-to-ANI ratio; the interaction between CNs and PANI was affected by the CNs-to-ANI ratio. Though the $\mathrm{N}^{*}$-LCs mesophase was unstable during the in situ polymerization of ANI, the goal of this work is to develop a promising method for in situ asymmetric polymerization of ANI in the $\mathrm{N}^{*}$-LCs of CNs and also to expand the possibility of application of nanocomposites of PANI/CNs. Obviously, many open questions such as stability and controllability of the helical sense and twisting power of the lyotropic $\mathrm{N}^{*}$-LCs and the possible influence of the $\mathrm{N}^{*}$-LCs used for preparing PANI/CNs should be answered experimentally and theoretically to fully understand the properties of the PANI/CNs nanocomposites.

\section{Conflict of Interests}

The authors declare that they have no conflict of interests.

\section{Acknowledgments}

The authors gratefully acknowledge Professor Tsunehisa Kimura and Dr. Fumiko Kimura (Kyoto University, Japan) for their helpful discussions and technical support. This work was partially supported by the Program for International S\&T Cooperation Projects of China (2011DFA50430), National Natural Science Foundation of China (50773033), Science Foundation of Shandong Province (Y2007F01), and Doctoral Fund of QUST.

\section{References}

[1] T. A. Skotheim, Handbook of Conducting Polymers, Conjugated Polymers, CRC Press, Boca Raton, Fla, USA, 3rd edition, 2007.

[2] D. L. Wise, Electrical and Optical Polymer Systems: Fundamentals, Methods, and Applications, Marcel Dekker, New York, NY, USA, 1998.

[3] A. J. Heeger, "Semiconducting and metallic polymers: the fourth generation ofpolymeric materials (Nobel Lecture)," Angewandte Chemie International Edition, vol. 40, pp. 2591-2611, 2001.

[4] T. Suga, H. Ohshiro, S. Ugita, K. Oyaizu, and H. Nishide, "Emerging N-type redox-active radical polymer for a totally organic polymer-based rechargeable battery," Advanced Materials, vol. 21, no. 16, pp. 1627-1630, 2009.

[5] K. Koshika, N. Sano, K. Oyaizu, and H. Nishide, "An ultrafast chargeable polymer electrode based on the combination of nitroxide radical and aqueous electrolyte," Chemical Communications, no. 7, pp. 836-838, 2009.

[6] G. Nyström, A. Razaq, M. Strømme, L. Nyholm, and A. Mihranyan, "Ultrafast all-polymer paper-based batteries," Nano Letters, vol. 9, no. 10, pp. 3635-3639, 2009. 
[7] K. Fleming, D. Gray, and S. Matthews, "Cellulose crystallites," Chemistry, vol. 7, pp. 1831-1835, 2001.

[8] D. Klemm, B. Heublein, H. P. Fink, and A. Bohn, "Cellulose: fascinating biopolymer and sustainable raw material," Angewandte Chemie-International Edition, vol. 44, no. 22, pp. 3358-3393, 2005.

[9] J. Sugiyama, R. Vuong, and H. Chanzy, "Electron diffraction study on the two crystalline phases occurring in native cellulose from an algal cell wall," Macromolecules, vol. 24, no. 14, pp. 41684175, 1991.

[10] X. M. Dong, J. F. Revol, and D. G. Gray, "Effect of microcrystallite preparation conditions on the formation of colloid crystals of cellulose," Cellulose, vol. 5, no. 1, pp. 19-32, 1998.

[11] S. Beck-Candanedo, M. Roman, and D. G. Gray, "Effect of reaction conditions on the properties and behavior of wood cellulose nanocrystal suspensions," Biomacromolecules, vol. 6, no. 2, pp. 1048-1054, 2005.

[12] S. Elazzouzi-Hafraoui, Y. Nishiyama, J. L. Putaux, L. Heux, F. Dubreuil, and C. Rochas, "The shape and size distribution of crystalline nanoparticles prepared by acid hydrolysis of native cellulose," Biomacromolecules, vol. 9, no. 1, pp. 57-65, 2008.

[13] Y. Habibi, L. A. Lucia, and O. J. Rojas, "Cellulose nanocrystals: chemistry, self-assembly, and applications," Chemical Reviews, vol. 110, no. 6, pp. 3479-3500, 2010.

[14] J. Pan, W. Hamad, and S. K. Straus, "Parameters affecting the chiral nematic phase of nanocrystalline cellulose films," Macromolecules, vol. 43, no. 8, pp. 3851-3858, 2010.

[15] K. Akagi, "Helical polyacetylene: asymmetric polymerization in a chiral liquid-crystal field," Chemical Reviews, vol. 109, no. 11, pp. 5354-5401, 2009.

[16] Z. L. Mo, Z. L. Zhao, H. Chen, G. P. Niu, and H. F. Shi, "Heterogeneous preparation of cellulose-polyaniline conductive composites with cellulose activated by acids and its electrical properties," Carbohydrate Polymers, vol. 75, no. 4, pp. 660-664, 2009.

[17] W. Yin, J. Li, Y. Li, Y. Wu, T. Gu, and C. Liu, "Conducting IPN based on polyaniline and crosslinked cellulose," Polymer International, vol. 42, no. 3, pp. 276-280, 1997.

[18] L. H. C. Mattoso, E. S. Medeiros, D. A. Baker, J. Avloni, D. F. Wood, and W. J. Orts, "Electrically conductive nanocomposites made from cellulose nanofibrils and polyaniline," Journal of Nanoscience and Nanotechnology, vol. 9, no. 5, pp. 2917-2922, 2009.

[19] X. Shi, L. Zhang, J. Cai et al., "A facile construction of supramolecular complex from polyaniline and cellulose in aqueous system," Macromolecules, vol. 44, no. 12, pp. 4565-4568, 2011.

[20] W. 1. Hu, S. Y. Chen, Z. H. Yang, L. T. Liu, and H. P. Wang, "Flexible electrically conductive nanocomposite membrane based on bacterial cellulose and polyaniline," Journal of Chemical Physics $B$, vol. 115, pp. 8453-8457, 2011.

[21] J. Stejskal, M. Spirkova, A. Riede, M. Helmstedt, P. Mokreva, and J. Prokes, "Polyaniline dispersions 8 . The control of particle morphology," Polymer, vol. 40, no. 10, pp. 2487-2492, 1999.

[22] J. Pecher and S. Mecking, "Nanoparticles of conjugated polymers," Chemical Reviews, vol. 110, no. 10, pp. 6260-6279, 2010.

[23] S. Dorey, C. Vasilev, L. Vidal, C. Labbe, and N. Gospodinova, "Ultrafine nano-colloid of polyaniline," Polymer, vol. 46, no. 4, pp. 1309-1315, 2005.

[24] S. Y. Park, M. S. Cho, and H. J. Choi, "Synthesis and electrical characteristics of polyaniline nanoparticles and their polymeric composite," Current Applied Physics, vol. 4, no. 6, pp. 581-583, 2004.
[25] F. Jiang, A. R. Esker, and M. Roman, "Acid-catalyzed and solvolytic desulfation of H2SO 4-hydrolyzed cellulose nanocrystals," Langmuir, vol. 26, no. 23, pp. 17919-17925, 2010. 

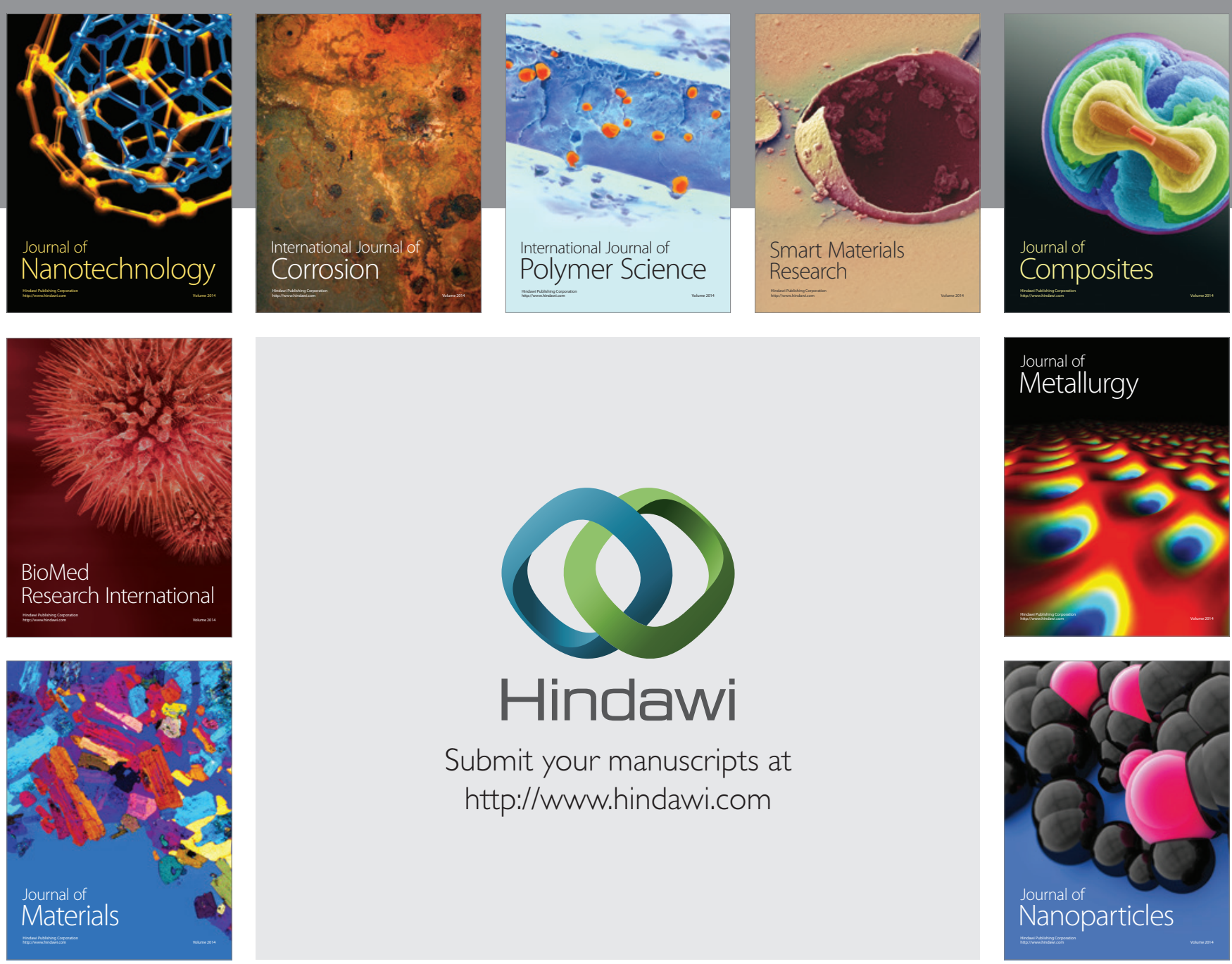

Submit your manuscripts at http://www.hindawi.com
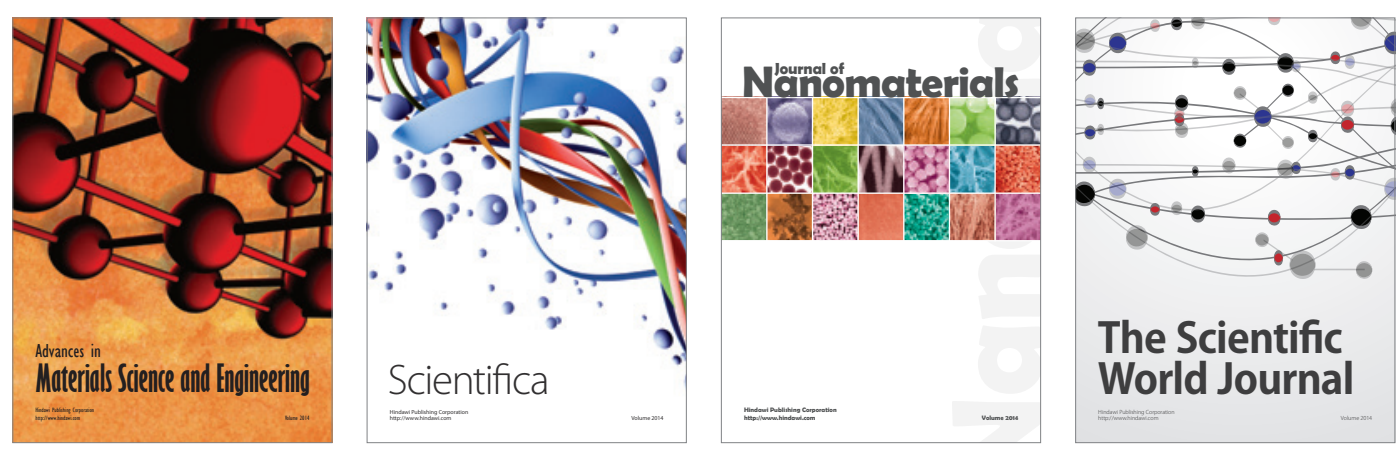

\section{The Scientific World Journal}
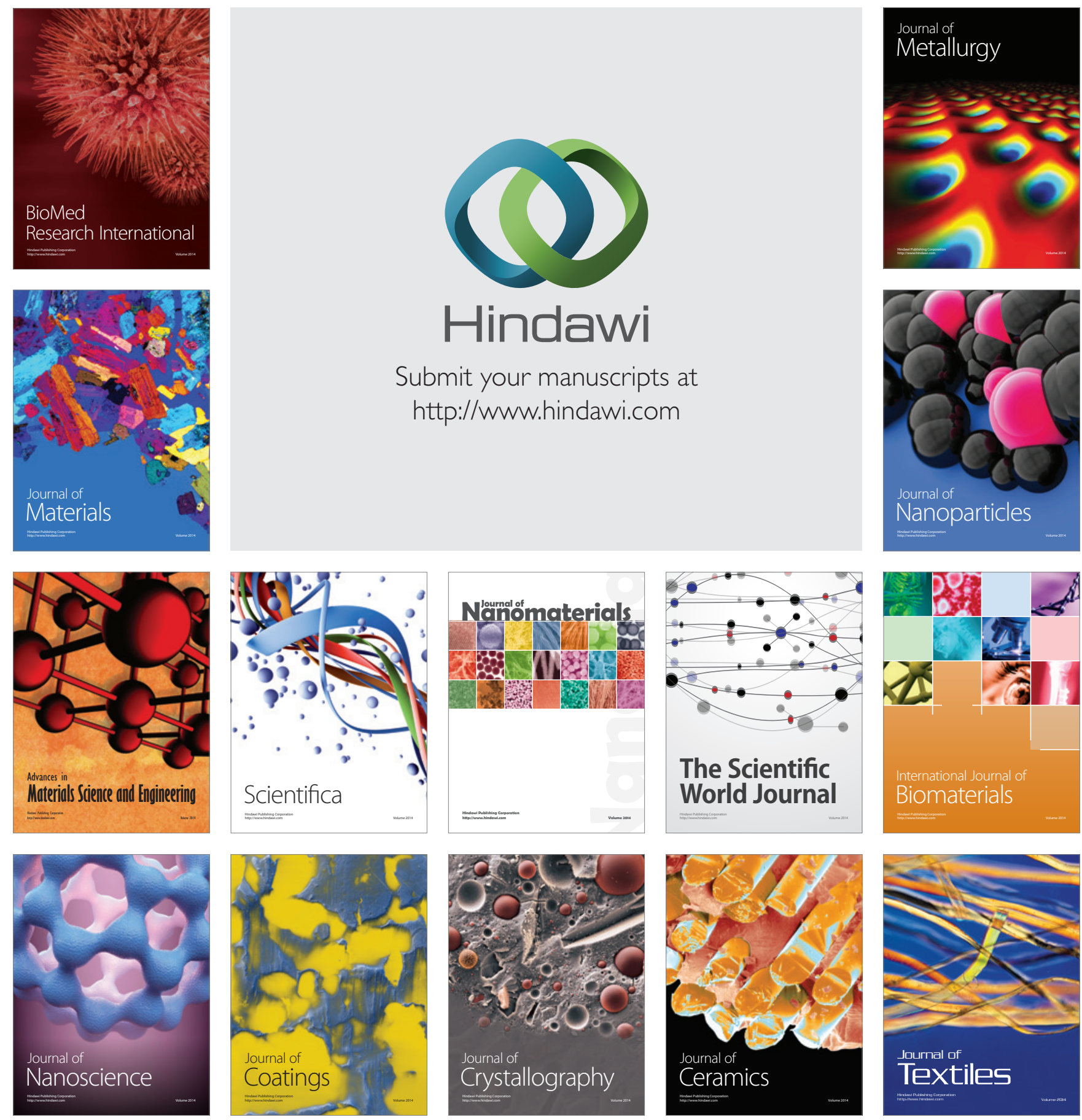